\title{
Robot Assisted Stapedotomy ex vivo with an Active Handheld Instrument*
}

\author{
Tobia Vendrametto, Jacob S. McAfee, Barry E. Hirsch, Cameron N. Riviere, Giancarlo Ferrigno, and \\ Elena De Momi
}

\begin{abstract}
Micron is a fully handheld active micromanipulator that helps to improve position accuracy and precision in microsurgery by cancelling hand tremor. This work describes adaptation, tuning, and testing of the Micron system for stapedotomy, a microsurgical procedure performed in the middle ear to restore hearing that requires accurate manipulation in narrow spaces. Two end-effectors, a handle, and a brace (or rest) were designed and prototyped. The control system was adapted for the new hardware. The system was tested ex vivo in stapedotomy procedure comparing manually-performed and Micron-assisted surgical tasks. Tremor amplitude was found to be reduced significantly. Further testing is needed in order to obtain statistically significant results regarding other parameters dealing with regularity of the fenestra shape.
\end{abstract}

Key words - medical robotics, tremor compensation, stapedotomy, handheld, micromanipulator.

\section{INTRODUCTION}

Otosclerosis is a hearing impairment due to abnormal and excessive bone growth. This interferes with the normal vibration of the middle ear ossicles, in particular the stapes, which is the final link in the transmission chain. Otosclerosis causes stapes fixation that restricts proper sound transmission to the inner ear, but it can be corrected by surgery [1].

Middle ear microsurgery is particularly challenging since surgeons manipulate human body parts about few hundred microns, and visualizing the surgical area through a microscope [2]. The surgery called stapedotomy [3], [4], consists in the replacement of the immobile stapes with a piston-prosthesis. After exposing the stapes, its superstructure is removed. Then a rosette (approximately circular shape made by overlapping laser bursts to weaken the bone and create an opening to house the prosthesis) on the stapes footplate is created, and a fenestra is opened. The rosette task requires high precision and accuracy and thus robotic assistance to stabilize hand motion, can be helpful. Subsequently, the piston-prosthesis is placed; it goes inside the fenestra and is secured to the incus. It replaces the

\footnotetext{
* Research supported by Politecnico di Milano and by the U.S. National Institutes of Health (grant no. R01EB000526).

T. Vendrametto is with the Department of Mechanical Engineering at Politecnico di Milano (phone: (+39) 340-0725162; e-mail: tobia.vendrametto@gmail.com ).

J. S. McAfee and B. E. Hirsch are with University of Pittsburgh Medical Center, Pittsburgh, PA 15213 USA.

C. N. Riviere is with the Robotics Institute, Carnegie Mellon University, Pittsburgh, PA 15213, USA.

G. Ferrigno and E. De Momi are with Department of Electronics, Information and Bioengineering, Politecnico di Milano, Milan, Italy.
}

immobile stapes and the normal vibration of the middle ear ossicles is restored.

In surgery, the most common robotic systems are teleoperated robots in which there is a mechanically grounded robotic arm with multiple links and a console to control the robot. These systems have several advantages, like tremor filtering, motion scaling and remote control, but they are also complex, bulky, expensive, and completely change the traditional surgical workflow [5].

To overcome these drawbacks, a novel handheld robot called "Micron" was first proposed by Riviere et al. [6], in order to cancel hand tremor and enhance positioning accuracy during microsurgery. Handheld robots have the advantage of being inexpensive compared with other surgical robotic systems, and easily integrated in the conventional surgical workflow, since they are small and similar to a surgical instrument. They are intuitive to use, since they are handheld, and they have a safety advantage, because if the system fails, removal from the surgical field is easy and the surgery can be completed with conventional tools [5].

Micron is a fully handheld micromanipulator that aims to cancel hand tremor and achieve high positioning accuracy [7]. Its main components are:

- Micron handpiece (Fig. 1): it compensates hand tremor. Three piezoelectric motors deflect the tip in 3DOF with a motion equal but opposite to the hand tremor, so that compensation is accomplished.

"ASAP" (Apparatus to Sense Accuracy of Position): its main components are two position-sensitive detectors and four infrared light-emitting diodes [8]. This apparatus detects the tool position in 6 DOF with a resolution of $4 \mu \mathrm{m}$ at $2000 \mathrm{~Hz}$.

- A real-time computer runs the control loop.

\section{Design Methods}

Micron was developed and adapted for middle ear surgery, taking as reference the demands of stapedotomy.

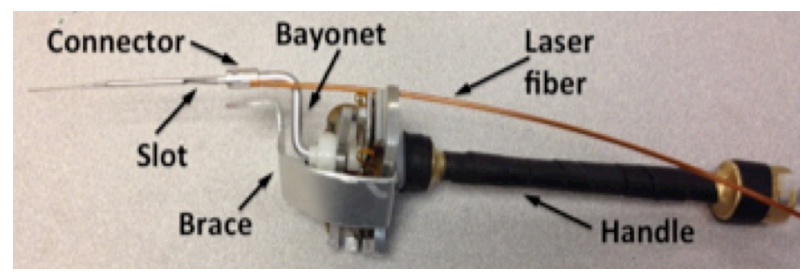

Fig. 1: Micron developed for middle-ear procedure: laser tool, brace and handle. The laser fiber comes from the laser machine and passes inside the laser probe through a slot, so the metal tip of the laser fiber is preserved. 


\section{A. Ear Model}

An artificial middle ear phantom was developed to test and assess dimensions and provide a working environment. It was made with dimensions according to [9]. The model incorporates an ear speculum, a plastic tube that resembles the ear canal, and a slip of thick paper on which a stapes footplate-like shape was printed (Fig. 2).

\section{B. Laser probe}

Starting from the OmniGuide, BeamPath OTO-S $\mathrm{CO}_{2}$ laser fiber, a Micron-mountable laser probe was built, as seen in Fig. 1. It was made with a bayonet-shaped mount for an improved visualization of the middle ear, a connector made of aluminum, and the laser probe, which was a hollow stainless steel tube with a slot on the side for the fiber to pass through. Its high stiffness prevented vibration and its light weight resulted in low inertia, so it could be easily moved by the three piezoelectric stacks.

\section{Brace and Handle}

Two components were designed: a brace and handle (Fig. 1), for better compatibility with stapedotomy than the previous version [10].

The handle was fabricated using 3D printing. Brass was used because it is cheap and allows small wall thickness ( $\min 0,5 \mathrm{~mm}$ ) during the 3D-printing process.

The other component developed was a brace, rigidly fixed to Micron base. It was essential for two reasons:

- Protecting the tool moved by the stacks. If the tool touches something, motors saturate, and cancellation no longer works;

- Providing a resting point for the user: since the brace is rigidly fixed, the user can rest it against the speculum instead of resting the tool shaft. This allows stabilizing the hand and thus is a first tremor-reduction technique.

\section{Control tuning}

Micron control was tuned to obtain the best tremor cancellation with the new hardware. Micron has internal model control (IMC) (control block diagram can be seen in [7]), so a dynamic model of the manipulator with new tools was needed. A system identification process was implemented. A sweep sine signal $(5-1000 \mathrm{~Hz})$ was injected. The response of the Micron system was collected and analyzed in the frequency domain. A black-box model approach was used and state-space models were found, focusing on the $10-500 \mathrm{~Hz}$ frequency range, using 14 and 12

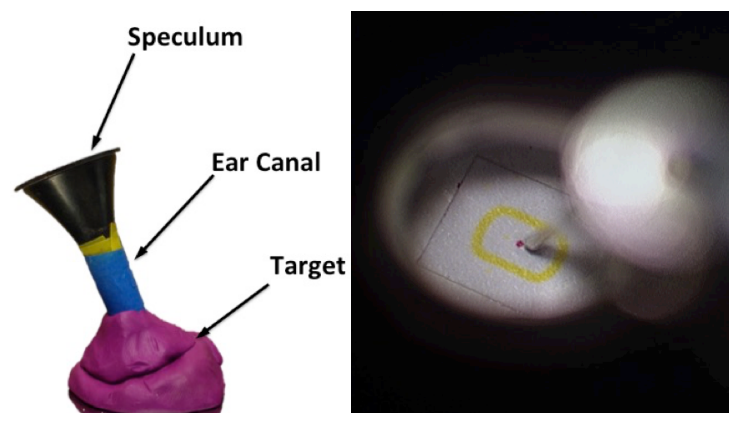

Fig. 2: Ear model (left) and view from the microscope (right).

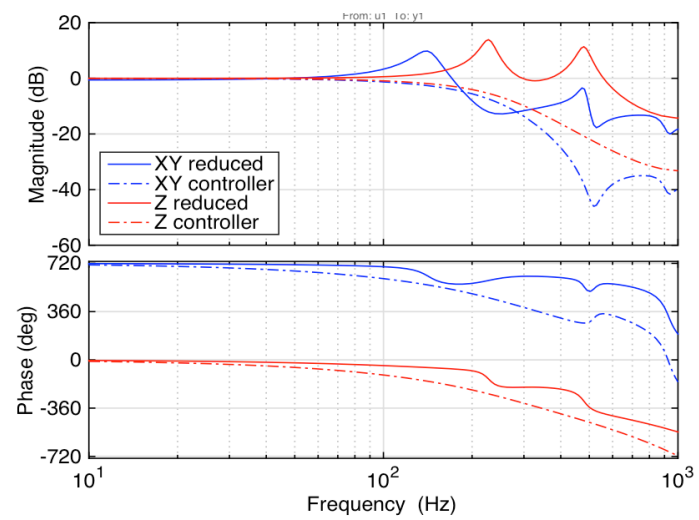

Fig. 3: Bode plot of the manipulator lateral ( $x y$, blue) and axial $(z$, red) dynamic models. The system-filtered responses (dotted lines) are shown as well. This low-pass response suppresses the instability peaks, but adds lag.

states for the lateral $(x y)$ and axial $(z)$ motion respectively.

The number of states was a trade-off between best fit and complexity. Discarding the low Hankel singular values, an order reduction was achieved, with 8 and 6 states, respectively. Inverting the reduced model, keeping only the stable poles and adding two pairs of low-pass Bessel filter poles, the filter was obtained. The model multiplied by the filter gives the final dynamic response of the system.

Fig. 3 shows the dynamic models of lateral and axial motion of Micron tip. Instability peaks are present when the control is excluded (open loop). These resonances are smoothened by applying a filter: the responses considering the controller (close loop) are shown in dotted lines. A better control of the system is achieved at the price of adding lag.

The IMC system had a control bandwidth of $150 \mathrm{~Hz}$, with a lag parameter of 7 samples. This information was obtained by injecting a step change into the Micron control and analyzing the time response in terms of overshoot and rise time.

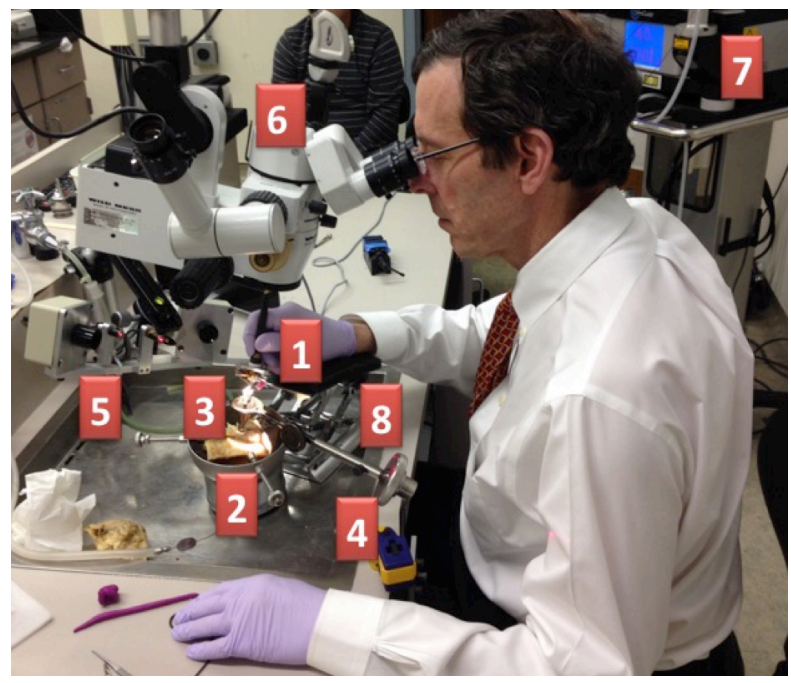

Fig. 4: Experiment setup: 1) Micron; 2) Bone and holder; 3) Speculum; 4) Speculum holder; 5) ASAP; 6) Microscope; 7) Laser machine; 8) Hand rest. 


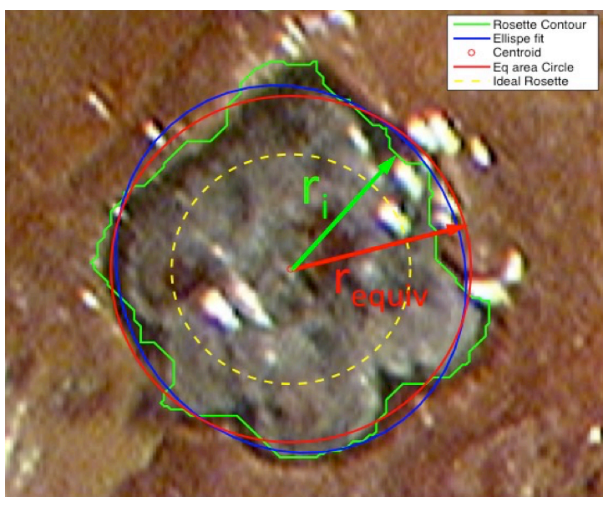

Fig. 5: Rosette contour detection. Contour (green), equivalent area circle (red) and best-fit ellipse (blue) are plotted.

\section{EXPERIMENTAL METHODS}

\section{A. Experiment description}

The rosette with subsequent fenestration and removal of footplate char are the most delicate steps of stapedotomy [11]. The aim of the experiments was to test if, with the help of Micron, tremor reduction and better rosette geometry could be obtained. Using Micron and the developed laser probe, the task was to perform the rosette.

Tests were carried out by two experienced otologic surgeons under a board-approved protocol, with robotic aid (Micron on) and unaided (Micron off). Five sessions were performed using the ear phantom. Subsequently, two sessions, using cadaver temporal bones, to test Micron in conditions closer to real surgery. The order of test conditions was varied to exclude order effects. For every session one aided and one unaided case were performed.

\section{B. Experimental Setup}

Fig. 4 shows the experimental setup. The surgeon held Micron, with the instrument inside the speculum. The laser probe was mounted on Micron, with the laser fiber coming from the laser machine, behind the surgeon. The speculum, fixed by a speculum holder (so a stable resting point was obtained) was inside the ear canal, giving access to the middle ear area. To magnify the working area a microscope (Leica Wild M650) was used with 16x or 25x magnification, according to surgeon's preference. To replicate surgical conditions, a hand rest was provided to simulate the patient's head. The ASAP was placed in front of the surgeon.

\section{Performance measures and analysis}

Excessive movement can lead to damage of middle ear structures. To quantify Micron performance, tremor characteristics and the regularity of the rosettes' shape were analyzed. Since the number of phantom and bone trials was small, all the data for the same variable were grouped together for analysis.

Analyzing the tip trajectory signal recorded during the rosette tasks, it was possible to estimate the tremor component by applying a band-pass ideal filter with bandwidth 6-12 Hz according to [12]. The tremor was characterized by two quantities: (i) $R M S_{\text {tremor }}$, RMS (root
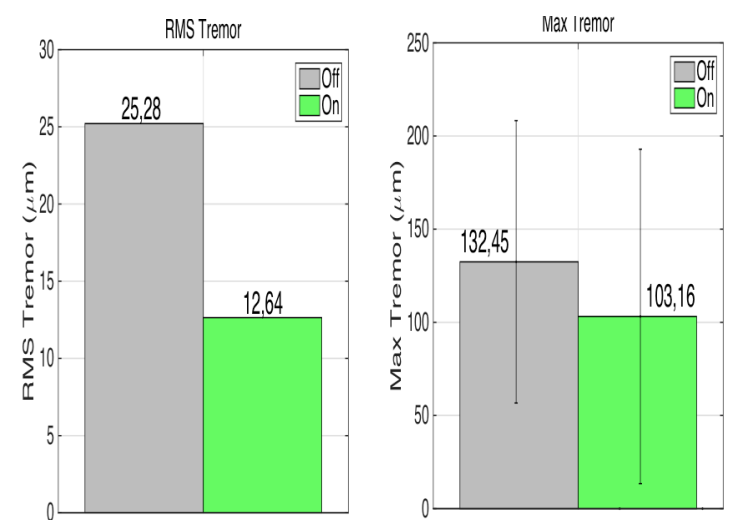

Fig. 6: Ensemble trials tremor data: $R M S_{\text {tremor }}$ (left) and average $\operatorname{Max}_{\text {tremor }}$ (right). Error bars indicate standard deviation.

mean square) calculated on the concatenated tremor signals from all trials:

$$
R M S_{\text {tremor }}=\sqrt{\sum_{i=1}^{m} d_{i}^{2} / m}
$$

where $d_{i}$ is the 3D displacement due to tremor (Fig. 7) and $m$ the total number of trajectory points; and (ii) Max ${ }_{\text {tremor }}$, maximum 3D tip displacement $\left(d_{i}\right)$ due to tremor during the rosette task, important in surgery because just one wrong motion can lead to important tissue damage.

To characterize the geometry of the rosette, it was necessary to detect its contour. A color-based segmentation method using K-means clustering was used to analyze the images from the microscope and detect the rosette contour [13]. Once the contour was detected, it was possible through MATLAB to compute the centroid, the best-fit ellipse, and the circle with equivalent area since the contour and the area were known, as seen in Fig. 5.

Two measures were chosen to characterize the rosette shape: (i) eccentricity $e$ of the best-fit ellipse, indicating how circular the rosette was; (ii) $R M S_{\text {contour }}, \mathrm{RMS}$ of the distance between the rosette contour $r_{i}$ and the equivalent-area circle $r_{\text {equiv }}$ (Fig. 5), indicating how irregular the rosette contour was:

$$
R M S_{\text {contour }}=\sqrt{\sum_{i=1}^{n}\left(r_{i}-r_{\text {equiv }}\right)^{2} / n}
$$

where $n$ is the total number of points in the contour.

Two non-parametric tests were used to determine statistical significance $(p<0,05)$ : Kolmogorov-Smirnov (KS) two-sample test and Kruskal-Wallis (KW) test. Nonparametric tests were chosen because they do not rely on parameters estimated form the distribution, since the data population was small and the distribution unknown.

\section{RESUltS}

\section{A. Tremor}

Fig. 6 shows the $R M S_{\text {tremor }}$ and Max tremor $_{\text {average during }}$ ensemble trials. The tremor reduction is noteworthy for both quantities: $-50.0 \%$ and $-22.1 \%$, respectively. $R M S_{\text {tremor }}$ is a single value computed on concatenated signals, so there is no standard deviation. For Max tremor the $\mathrm{KS}$ and $\mathrm{KW}$ tests gave contrasting results in terms of $p$-value, but according to 


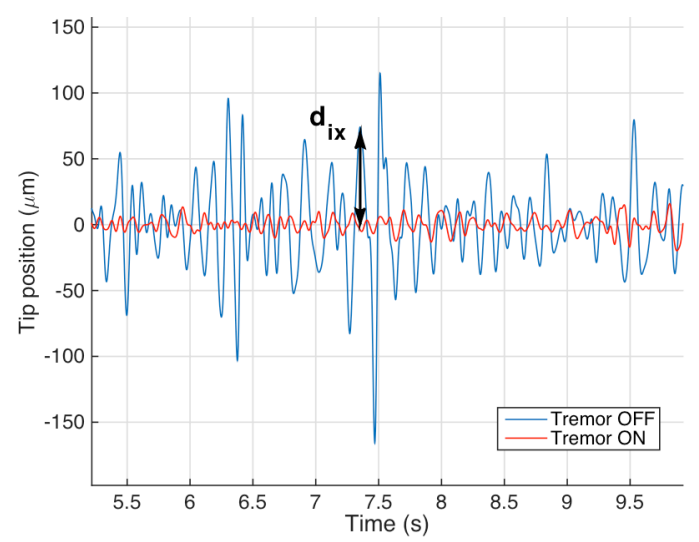

Fig. 7: Tremor cancellation effect: tremor amplitude in unaided case (blue) and aided case (red). To show Micron effect, 1D motion along the $\mathrm{x}$-direction is shown, but the tremor is $3 \mathrm{D}$, so $\mathrm{x}, \mathrm{y}$ and $\mathrm{z}$ component were considered. The $\mathrm{x}$ displacement component $d_{i x}$ is shown: this is the displacement from the zero-line to the tremor signal line.

the high standard deviation statistical significance seems dubious. Fig. 7 shows typical results in tremor cancellation.

\section{B. Rosette geometry}

Fig. 8 shows the mean across all trials of eccentricity $e$ and $R M S_{\text {contour }}$ error with respect to a circle of equivalent area. It can be seen that $e$ is slightly reduced in the aided case. The $R M S_{\text {contour }}$ decreases when Micron is on; however, the difference is less than $10 \mu \mathrm{m}$. Statistical significance was not reached in these cases.

\section{DISCUSSION}

In terms of tremor, Micron cancellation is effective, since $R M S_{\text {tremor }}$ is reduced, resulting in a more controlled tip motion and reduced risk to hurt delicate middle ear tissues. $M_{\text {ax }}$ tremor, $e$ and $R M S_{\text {contour }}$ are reduced, but these results are not confirmed by statistics. Nevertheless other factors affect the result when Micron is used, such as surgeon's fatigue, amount of training with Micron, and experience level. These factors need to be further investigated to assess their influence on outcomes, i.e., analyzing the learning curve.

Surgeons' opinion highlights the potential to control fine movements to minimize undesired excursions and the
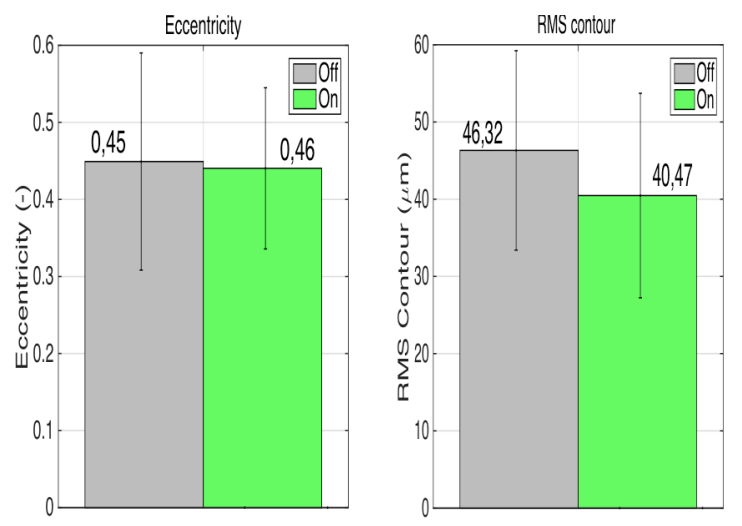

Fig. 8: Ensemble trials rosette geometrical data: average eccentricity (left) and average $R M S_{\text {contour }}$ (right). Error bars indicate standard deviation. possible use of Micron in other surgical fields (e.g. ophthalmology). Complains were the hindering of natural motion that sometimes happened due to saturation. To overcome this limitation the interaction between speculum, tool and motors need to be analyzed and improved in order to overcome saturation.

The results indicate that further experimentation is necessary in order to obtain significant results with regard to regularity of the rosette. Vision-based virtual fixtures [14] may be needed in order to improve laser targeting accuracy. Virtual fixtures could also enhance safety by preventing penetration of the vestibule during removal of char fragments from the fenestra after laser application.

\section{ACKNOWLEDGMENTS}

Thanks to the members of the Surgical Mechatronics Laboratory: Nate Wood, Trent Wells, Craig Lehocky, and in particular Sungwook Yang and Rob MacLachlan. Thanks to Linda Phillips of OmniGuide for providing the laser.

\section{REFERENCES}

[1] R. R. F. Ruby and R. H. Ballagh, "Reconstructive middle ear surgery." Can. Fam. Phys., pp. 2689-2693, 1992.

[2] S. Charles et al. , "Dexterity-enhanced Telerobotic Microsurgery," In Proc. Int. Conf. Adv. Robot., 1997, pp. 5-10.

[3] R. S. Haberman II, Middle Ear and Mastoid Surgery. New York: Thieme, 2004.

[4] J. J. Shea Jr, "Forty years of stapes surgery," Am. J. Otol., vol. 19, pp. 52-5, 1998.

[5] C. J. Payne and G.-Z. Yang, "Hand-Held Medical Robots," Ann Biomed. Eng., vol. 42, no. 8, pp. 1594-1605, Jun. 2014.

[6] C. N. Riviere and N. V Thakor, "Modeling and canceling tremor in human-machine interfaces," IEEE Eng. Med. Biol. Mag., vol. 15, no. 3, pp. 29-36, 1996.

[7] R. A. MacLachlan, B. C. Becker, J. Cuevas Tabares, G. Podnar, L. A. Lobes, Jr., and C. N. Riviere, "Micron: an actively stabilized handheld tool for microsurgery," IEEE Trans. Robot., vol. 28, no. 1, pp. 195-212, 2012.

[8] R. A. MacLachlan and C. N. Riviere, "High-speed microscale optical tracking using digital frequency-domain multiplexing," IEEE Trans. Instrum. Meas., vol. 58, no. 6, pp. 1991-2001, Jun. 2009.

[9] M. Miroir, Y. Nguyen, J. Szewczyk, O. Sterkers, and A. Bozorg Grayeli, "Design, kinematic optimization, and evaluation of a teleoperated system for middle ear microsurgery,"

ScientificWorldJournal., vol. 2012, p. 907372, Jan. 2012.

[10] G. Montes Grande, A. J. Knisely, B. C. Becker, S. Yang, B. E. Hirsch, and C. N. Riviere, "Handheld micromanipulator for robotassisted stapes footplate surgery," Conf Proc IEEE Eng Med Biol Soc, vol. 2012, pp. 1422-5, 2012.

[11] D. L. Rothbaum, J. Roy, D. Stoianovici, P. Berkelman, G. D. Hager, R. H. Taylor, L. L. Whitcomb, H. W. Francis, and J. K. Niparko, "Robot-assisted stapedotomy: micropick fenestration of the stapes footplate," Otolaryngol Head Neck Surg, vol. 127, no. 5, pp. 417-426, 2002.

[12] T. S. Wells, S. Yang, R. A. MacLachlan, J. T. Handa, P. Gehlbach, and C. Riviere, "Comparison of baseline tremor under various microsurgical conditions," in Proc. IEEE Int. Conf. Syst. Man Cybern., 2013, pp. 1482-7.

[13] MathWorks, "Color-Based Segmentation Using K-Means Clustering." [Online]. Available:

http://it.mathworks.com/help/images/examples/color-basedsegmentation-using-k-means-clustering.html.

[14] B. C. Becker, R. A. MacLachlan, L. A. Lobes, G. D. Hager, and C. N. Riviere, "Vision-based control of a handheld surgical micromanipulator with virtual fixtures," IEEE Trans. Robot., vol. 29, no. 3, pp. 674-683, Jun. 2013. 